TITLE:

\title{
Dynamic characteristics and dual control of a ball screw drive with integrated piezoelectric actuator
}

\section{$\operatorname{AUTHOR}(\mathrm{S})$ :}

Fujita, Tomoya; Matsubara, Atsushi; Kono, Daisuke; Yamaji, Iwao

\section{CITATION:}

Fujita, Tomoya ...[et al]. Dynamic characteristics and dual control of a ball screw drive with integrated piezoelectric actuator. Precision Engineering 2010, 34(1): 34-42

\section{ISSUE DATE:}

2010-01

URL:

http://hdl.handle.net/2433/123372

\section{RIGHT:}

Copyright @ 2009 Elsevier; この論文は出版社版でありません。引用の 際には出版社版をご確認ご利用ください。; This is not the published version. Please cite only the published version. 
Type of contribution : Original research papers

Title:

Dynamic Characteristics and Dual Control of a Ball Screw Drive with Integrated Piezoelectric Actuator

\author{
Tomoya FUJITA ${ }^{1}$, Atsushi MATSUBARA ${ }^{1}$, Daisuke KONO $^{1}$, Iwao YAMAJI ${ }^{1}$ \\ ${ }^{1}$ Department of Micro Engineering, Graduate School of Engineering, Kyoto \\ University, Yoshida-honmachi, Sakyou-ku, Kyoto, 606-8501, Japan
}

Mailing name and address: Atsushi MATSUBARA; room713, Department of Micro Engineering, Graduate School of Engineering, Kyoto University, Yoshida-honmachi, Sakyou-ku, Kyoto, 606-8501, Japan

Telephone: +81-75-753-5863

Facsimile: $+81-75^{-771-7286}$

E-mail: matsubara@prec.kyoto-u.ac.jp

\begin{abstract}
This paper describes the precision continuous path tracking control by using a dual-actuated single stage. First, fine-drive mechanism and the dynamic model of the entire drive system are described. In the simulation model, the dynamic characteristic of the dual-actuated stage is investigated to see whether it can provide precise motion by using dual control. Second, the fine motion controller is designed. Adjusting the control parameters, a positioning resolution of $20 \mathrm{~nm}$ and a bandwidth of $260 \mathrm{~Hz}$ were obtained. Third, the frequency responses of coarse and fine drives are experimentally investigated. After that, the dual controller is designed based on the investigated dynamics. Finally, whether coarse-motion and fine-motion could work complimentarily by the dual servo is examined in the experiments. By using the simultaneous dual controller, tracking errors were reduced sufficiently compared to the single coarse control.
\end{abstract}

Keywords: Precision positioning, Dual servo control, Ball screw drive, Piezoelectric actuator 


\section{Introduction}

In recent years, demands for high-precision machine tools are increasing in the manufacturing fields of precision dies and molds, optical parts, and high precision components for electrical parts. For such high-precision machines, drive systems with sub-micrometer accuracy over a travel range of several hundred millimeters are necessary. Conventional ball-screw drives have versatility to respond to various applications, but it is difficult to achieve high-precision motion without special design techniques.

One approach to improving motion accuracy of the drive systems is to introduce linear motors (LMs). Futami et al. developed a nanometer positioning system by using a LM [1]. Pritshow reported a great potential of LMs as high speed and high response actuators [2]. After inspired by these early works, the application of LMs has been expanding; and there are many kinds of LM machines in today's market of machine tools [3].

While having high response that contributes to enhancing motion accuracy, the linear motor drives suffer from the following drawbacks: it is difficult to obtain high load capacity and high stiffness in compact design; gravity cancellation mechanism is required on vertical axes; the feedback signals detected by position sensors such as linear encoders are sensitive to structural vibrations. Hsieha et al. designed a dual linear-motor drive with a pneumatic gravity compensator for the vertical-axis drive of a high-speed EDM [4]. Weck et al. discussed the influence of machine vibrations on the feedback control of a linear motor [5]. These literatures implies that applying LMs to high-precision machine tools that have more than 3 axes requires optimal design techniques that take the structure dynamic and gravity into account.

Another approach to obtaining high positioning accuracy over the long travel range is to use dual servo systems. A typical design employs a dual-stage, stacking a fine motion stage on a coarse motion stage (global stage), which is widely used in many applications [6-10]. Moriyama et al. developed an X-Y stage for step and repeat type lithography systems, which comprises of a coarse stage driven by DC servomotors and ball-screws and a fine stage driven by piezoelectric actuators (PAs) [6]. Lee and Kim developed a dual stage for the alignment of wafers in microlithography [7]. Their system has a linear-motor-driven (global) stage and a PA-driven (fine) stage, which is capable of positioning the table with nanometer accuracy over the $200 \mathrm{~mm}$ travel distance. One important consideration in the dual-stage design is that it requires much space. An offset between the coarse-motion and fine-motion axes is another consideration, which makes it difficult to determine how to detect the table position. These considerations are crucial in the design of machine tools, as the usability and accuracy should be balanced. 
Another mechanical design for dual servo systems is based on the integration of fine and coarse actuators, and the designed feed drive have a dual-actuated single stage. Chen built a PA in the double nuts of a ball-screw so that it can control the preload of the nuts as well as the fine motion of the table [11]. Otsuka developed a small stage that has a PA between the support-bearing unit and the base unit [12]. In this table, one-nanometer positioning resolution was achieved with the combination of a high-resolution linear encoder. The both mechanical designs can provide high stiffness as the drive tables are mainly supported by rigid guideways that can prevent external moments from directly applying on the PA. However, the stiffness in the pulling direction of PA is not assured in Otsuka's design; the integration of the PA and the tuning of the preload are not easy in Chen's design.

To cope with these problems, Tanaka developed a fine positioning device that has a PA integrated in the support-bearing unit of a ball screw [13]. Since the PA provides not only fine motions to the table through the screw-supporting system but also preloads to support bearings in Tanaka's design, high table stiffness is expected with regard to pulling forces to the PA. Tanaka's fine positioning device achieves five-nm-step positioning with the high resolution linear encoder feedback. Since his research object is to built a Point-to-Point (PTP) positioning system, switching control of fine and coarse motion is adopted in his research. In the switching control, coarse motion is activated first, then switched to fine motion when table position approaches the target position within the certain distances that fine motion can cover. For such PTP positioning, it is possible to implement a stepping motor as the coarse actuator without the detection of the rotary angle.

In our work, to achieve high accuracy Continuous-Path (CP) tracking, we redesigned the Tanaka's device by changing the stepping motor to a high response AC servo motor with a rotary encoder to avoid the vibration problem caused by step pulses. The dynamic characteristic of the developed dual-actuated single stage is investigated to see whether it can provide precise CP tracking by using dual control. First, the fine-drive mechanism and the dynamic model of the entire drive system are described. Second, the frequency responses of coarse and fine drives are experimentally investigated. Then, simultaneous dual controller is designed based on the analysis of the fine and coarse motion mechanism. Finally, whether coarse-motion and fine-motion could work complimentarily by the dual servo is examined in the experiments. 


\section{Ball screw drive with integrated piezoelectric actuator \\ 2.1 Specification of the developed system}

Figure 1 shows a dual-actuated single stage feed drive developed for the investigation. Table 1 shows the specification of this system. The coarse motion is provided by the ball screw coupled with an AC servo motor. The screw-supporting system employs the following ball bearings: a deep-groove radial bearing that supports one screw-shaft end and moves axially toward the housing unit; a double-row angular-contact bearing in DF arrangement that supports the other screw-shaft end.

Figure 2 shows the details of the assembly of the fine motion mechanism. The angular-contact bearing has an inner spacer between the inner rings and a clearance between the outer rings. A stacked-type piezoelectric actuator is built between the end cap (preload cap) and the outer ring so that it can provide preloads to the bearing. Since this fine motion mechanism can be installed by simply modifying the support unit of the ball screw drive system, it can save space compared to the dual stage, which lead to the reduction of the Abbe's error.

\subsection{Drive mechanism of fine motion}

Figure 3 shows how the PA moves the table. Through the spacer, one of the outer rings is pushed by piezoelectric actuator, which causes inequality of the forces between two bearings (Fig. 3(b)). Then, inner races of the bearings and the screw shaft move to balance the forces between bearings. Finally, the table and the ball screw move together (Fig. 3(c)). 


\section{Open-loop frequency response and dynamic model}

\subsection{Dynamic model of the system}

In order to analyze the dynamic characteristic, the entire drive system is modeled by using a lumped parameter system shown in Fig. 4. In this model, the rigidity of the PA is assumed to be infinite.

\section{Nomenclature}

$J_{m}$ : Inertia moment of the motor $\left[\mathrm{kg} \cdot \mathrm{m}^{2}\right]$

$J_{b}$ : Inertia moment of the ball screw $\left[\mathrm{kg} \cdot \mathrm{m}^{2}\right]$

$m_{t}$ : Table mass $[\mathrm{kg}]$

$m_{b \text { : }}$ Ball screw mass [kg]

$K_{b s}$ : Axial stiffness of the ball screw shaft $[\mathrm{N} / \mathrm{m}]$

$K_{n}$ : Axial stiffness of the nut of the ball screw $[\mathrm{N} / \mathrm{m}]$

$K_{b}$ : Axial stiffness of the support bearing of the ball screw $[\mathrm{N} / \mathrm{m}]$

$K_{g}$ : Torsional stiffness of the ball screw [N/m]

$D_{m}$ : Coefficient of rotational viscous damping of the motor bearing $[\mathrm{N} \cdot \mathrm{m} \cdot \mathrm{s} / \mathrm{rad}]$

$D_{b}$ : Coefficient of rotational viscous damping of the support bearing of the ball screw $[\mathrm{N} \cdot \mathrm{m} \cdot \mathrm{s} / \mathrm{rad}]$

$C_{b}$ : Coefficient of viscous damping of the support bearing of the ball screw $[\mathrm{N} \cdot \mathrm{s} / \mathrm{m}]$

$C_{t}$ : Coefficient of viscous damping of the guide way of the table $[\mathrm{N} \cdot \mathrm{s} / \mathrm{m}]$

$T:$ Motor torque $[\mathrm{N} \cdot \mathrm{m}]$

$\theta_{m}$ : Rotating angle of the motor [rad]

$\theta_{b}$ : Rotating angle of the ball screw [rad]

$X_{p}$ : Displacement of the PA [m]

$X_{b c}$ : Displacement of the center of the angular-contact bearing [m]

$X_{s}$ : Axial displacement of the ball screw [m]

$X_{t}$ : Table displacement [m]

$V_{r}$ : Applied voltage to the PA [V]

$V_{\mathrm{c}}$ : Command voltage to the piezoamplifier[V]

It is known that the relationship between the command voltage and the displacement of the PA has nonlinear characteristics. Figure 5 shows the measured relationship of the PA used in our study. 


\subsection{Experimental setup of the system}

As the developed drive system is designed for a vertical axis of the high precision machine tool [14], all experiments are carried out by setting this system in the $\mathrm{Z}$ (vertical) direction as shown in Fig. 6.

A PC sends the torque command to a servo amplifier and the voltage command to a piezoamplifier through a 12-bit DA converter. The servo amplifier controls the motor torque with the current feedback. The piezoelectric amplifier magnifies the command voltage by 30 times, which is provided to the PA. A rotary encoder attached to the servo motor shaft and a linear encoder attached to the table detect the angle of the motor and the table position respectively, and the detected signals are sent to PC via a 32-bit counter board.

\subsection{Static and dynamic characteristic of the fine motion mechanism}

First, the static response of the fine motion mechanism is investigated. The table displacement, $X_{t}$, is simulated for the applied voltage to the PA, $V_{r}$. Figure 7 shows the commanded voltage for the simulation. In our study, the $V_{r}-X_{p}$ relation of the PA itself (shown in Fig. 5) is recorded as a data map and used as the input of the model shown in Fig.4. Figure 8 shows the simulated relationship between $V_{r}$ and $X_{t}$.

Comparing Fig. 5 and Fig. 8, it is observed that $X_{t}$ is identical to the half of $X_{p}$ in the simulation model. This is because the bearing center, $X_{b c}$, moves the half of $X_{p}$ and there are no other spring components that resist the moving elements (the screw shaft and the table).

To check whether the simulated fine motion is obtained, the same applied voltage is given to the PA and table displacement is measured with a capacitive type displacement sensor. Figure 9 shows the measured relationship between $V_{r}$ and $X_{t}$. It is found that the measured $X_{t}$ is $60 \%$ of the simulated $X_{t}$. This result implies the existence of spring and/or friction components that resist the motion of the PA.

Next, the frequency response is measured to investigate the dynamic characteristics. The command voltage for the measurement employs sinusoidal sweep signal, which has frequency ranges from $0.1-1000 \mathrm{~Hz}$ with three different amplitudes. Figure 10 shows the measured frequency responses from the command voltage to the table displacement. The resonance peaks was observed at $400-430 \mathrm{~Hz}$, which shifts slightly due to the amplitude change. In the low frequency range, the gain becomes large as the amplitude becomes large. These results imply that soft-spring characteristic exists in the fine motion mechanism. This may come from the nonlinear characteristics of the PA itself shown in Fig. 9, but it is difficult to prove it as the fine motion mechanism has other nonlinear components such as the balls of the bearings and the nut. 


\subsection{Dynamic characteristic of the coarse motion mechanism}

The dynamics of the coarse motion mechanism is investigated to check the effect of the implementation of the PA in the support bearing unit. The frequency response is calculated by using the model shown in Fig. 4. Figure 11 shows the simulated frequency response from the motor torque to the angular velocity. In the simulated response two sets of a resonance peak and an anti-resonance peak valley is observed, where the first set corresponds to the axial vibration mode, the second set the torsional vibration mode in the ball screw drive system. The resonance frequencies of the axial and torsional vibration are $500 \mathrm{~Hz}$ and $2000 \mathrm{~Hz}$ respectively. Both of the resonance frequencies are high enough to design feedback controllers.

To confirm the simulated result, the frequency response of the coarse motion mechanism is measured. Sinusoidal sweep signal is provided to the servo amplifier of the motor as torque command, and the angular velocity of the servo motor is measured. From the measured data, the frequency response is calculated. Figure 12 shows the measured frequency response from the motor torque to the angular velocity. The measured frequency response has almost the same gain-phase pattern as the simulated one. The resonance frequencies are $400 \mathrm{~Hz}$ for the axial vibration and $1800 \mathrm{~Hz}$ for the torsional vibration, both of which are smaller than the simulated ones. Even though installation of the fine motion mechanism lowers the natural frequencies of the ball screw drive, the effect is not significant for the feedback control. From these results, a conventional cascade type controller is employed for our study, which is shown in chapter 5 . 


\section{Fine motion control}

\subsection{Controller design and parameter adjustment of fine servo}

For the control law of the single fine motion, a PI + feedforward (FF) controller shown in Fig. 13 is employed. The linear encoder that detects the table position is used as the feedback signal. The control parameters are P-gain, $K_{p f}$, I-gain, $K_{i f}$ and feedforward (FF) gain, $K_{f f} \cdot G_{m f}(s)$ is the transfer function from the command voltage to the table position. As shown in Fig. 10, $G_{m f}(j \omega)$ looks like the frequency response of the second order system and its resonance frequency has a small sift. Hence, a notch filter is designed to suppress the resonance peak. The transfer function of the notch filter is as follows:

$G_{n}(s)=\frac{s^{2}+2 \varsigma_{n} \omega_{n} s+\omega_{n}{ }^{2}}{s^{2}+2 \varsigma_{d} \omega_{d} s+\omega_{d}{ }^{2}}$

, where $\omega_{n}$ and $\omega_{d}$ decide notch frequency: $\zeta_{n}$ and $\zeta_{d}$ decide the depth and width of the notch. To compensate the resonance peak, these parameters are tuned experimentally. The tuned parameters are as follows:

$\omega_{n}=350 \mathrm{~Hz}, \omega_{d}=350 \mathrm{~Hz}, \varsigma_{n}=\varsigma_{d}=0.3$.

By referring the frequency response of the fine motion mechanism with notch filter, $G_{n}(j \omega) G_{m f}(j \omega), K_{p f}$ is decided to be 0.20 so that servo loop has the appropriate gain margin. Then, $K_{i f}$ and $K_{f f}$ are decided to be 350 and 0.50 , respectively. Using these parameters, a bandwidth of $260 \mathrm{~Hz}$ is obtained in the dynamic response of the single fine servo from $X_{c f}$ to $X_{t}$.

\subsection{Step positioning test of the fine motion}

The step positioning experiment of the fine servo is carried out. The step widths of the command are $1 \mu \mathrm{m}, 500 \mathrm{~nm}, 100 \mathrm{~nm}$ and $20 \mathrm{~nm}$. The measurement was repeated three times in each case. Figure 14 shows the measured table displacement (detected with the linear encoder). Clear steps are observed in the table responses to input step width. Figure 15 shows the tool tip displacement measured with the capacitance displacement sensor. Clear steps are also observed in the tool tip response to input step width of $1 \mu \mathrm{m}, 500 \mathrm{~nm}$ and $100 \mathrm{~nm}$. Even though fluctuations are significant, steps are still seen in the response to the input step width of $20 \mathrm{~nm}$. The displacements measured at the table and tool tip have good agreements. This indicates the pitch motion of the table is small. This is because the driving center of the fine motion is close to the sensing line of the linear encoder and the distance between the sensing line and the tool tip point (Abbe's error) is small. According to these fundamental designs, the table displacements measured at the table can be assumed to be displacements at the tool tip. 


\section{Dual control of fine and coarse motion \\ 5.1 Dual controller design}

In order to control the coarse motion mechanism and the fine motion mechanism simultaneously, a dual controller is designed. The block diagram of the designed controller is shown in Fig. 16. In this figure, $X_{c}$ is the commanded position, $X_{\text {cofs }}$ is offset voltage of the fine motion, $l_{p}$ is the lead of the ball screw. The dual controller consists of a coarse control block and a fine motion control block. The coarse control block employs a cascade type controller with a velocity feedforward. The feedback control has PI-velocity loop and P-position loop. $K_{v f}, K_{v i}, K_{v p}$ and $K_{p p}$ are feedforward gain, I-gain of the velocity loop, P-gain of the velocity loop and P-gain of the position loop, respectively.

The motor position $\frac{l_{p}}{2 \pi} \theta_{m}$ is used for the feedback signal of the coarse motion loop. The reason why semi-closed loop control is employed is that $K_{p p}$ of the semi-closed loop could be higher than that of full-closed loop. In semi-closed system bandwidths of the velocity loop and position loop could be $150 \mathrm{~Hz}$ and $25 \mathrm{~Hz}$.

Position command, $X_{c}$, is forwarded to both coarse and fine motion control block. This means the coarse and the fine motion control is activated simultaneously, which is different from the conventional switching control of the fine motion and the coarse motion. Though this simultaneous dual control is a simple control scheme for CP tracking, the saturation of the command voltage for the fine motion is a critical issue. This is because the feedforward of the reference position is necessary for the control of the PAs. However, if the reference position is larger than the maximum travel distance of the fine motion mechanism, the command voltage for the fine motion saturates.

For the fine motion to track without such saturation over the travel distance, the deviation of the motor position and the table position is forwarded to the command voltage to the piezoamplifier. Another measure to the limitation of the PA displacement is that the command of the coarse motion is reduced by $X_{\text {cofs. }}$ This allows the fine motion to be both plus and minus where the table is located at the commanded position

The positioning error due to the thermal expansion of the ball screw can be also compensated by the fine motion. However, if the thermal expansion error is larger than the travel distance of the fine motion, fine motion control can’t compensate it. 


\subsection{Dual control experiment}

In order to evaluate the performance of simultaneous dual controller, the single coarse controller (semi-closed loop controller) and the dual controller were compared. The command trajectories are sinusoidal waves with amplitudes of $100 \mu \mathrm{m}$ and $500 \mu \mathrm{m}$. The period of the each sinusoidal wave is $10 \mathrm{~s}$.

Figure 17 depicts the tracking results for sinusoidal wave with amplitude of $100 \mu \mathrm{m}$. Figure 17 (a) and (b) show the table position and tracking errors with the single coarse motion controller, respectively. Figure 17(c) and (d) show the table position and tracking error with the dual controller, respectively. Obviously, the performance of the dual controller is better than the single coarse controller. The maximum tracking error with the single coarse controller is $2.5 \mu \mathrm{m}$. In addition, the motion error caused by the stick motion is observed in Fig.17 (b). On the other hand, the maximum tracking error with the dual controller is about $0.2 \mu \mathrm{m}$. Consequently, the dual control reduced the tracking error caused by the stick motion.

Figure 18 depicts the tracking results for sinusoidal wave with amplitude of $500 \mu \mathrm{m}$. As shown in Fig. 18 (b), the maximum tracking error with the single coarse controller is $6.5 \mu \mathrm{m}$. On the other hand, as shown in Fig.18 (d), the maximum tracking error with the dual controller is $2.5 \mu \mathrm{m}$. There still remains the tracking error caused by the stick motion with the dual controller. This is because the tracking error caused by the peak value of the stick motion was larger than the travel of the fine motion. 


\section{Conclusions}

This paper described the precision tracking control by using the dual-actuated single axis stage. This stage has a fine-drive mechanism with a piezoelectric actuator integrated into the support-bearing unit of a ball screw drive. The conclusions of this paper are summarized as follows:

(1) In the fine-drive mechanism, the PA pushes the outer ring of the support bearing unit, and this motion is transferred to the table. The table displacement is the half of the PA displacement in the simulation and one-third in the experiment. Hysterisis is observed in the relationship between the applied voltage of the PA and table displacement.

(2) The pitch motion of the table is small. This is because the driving center of the fine motion is close to the sensing line of the linear encoder and the distance between the sensing line and the tool tip point (Abbe's error) is small. According to these fundamental designs, the table displacement measured at the table can be assumed to be displacement at the tool tip.

(3) As the fine motion mechanism has also preload function, the natural frequencies of the entire drive system reduce little compared to those of the conventional ball screw drive system. The fine motion unit is modeled as a second order system, and PI position controller with a notch filter and a feedforward controller is designed. As a result, the positioning performance with a bandwidth of $230 \mathrm{~Hz}$ and a resolution of $20 \mathrm{~nm}$ is obtained for single fine servo.

(4) In order to control the coarse motion mechanism and the fine motion mechanism simultaneously, a dual feedback controller with the direct forward block of the deviation of the motor position and the table position to the piezoamplifier is designed. Experiments on tracking for the sinusoidal wave command were carried out. From the results, it was found that the maximum tacking error was reduced to $0.2 \mu \mathrm{m}$ for the command with an amplitude $100 \mu \mathrm{m}$. 


\section{Acknowledgement}

This research is partly supported by JSPS/MEXT Grant-in-Aid for Scientific Research (C) 18560103. The design and fabrication of the experimental device are supported by NSK Ltd., Mitsubishi Electric Corporation, and HEIDENHAIN K.K.. Authors would like to give special thanks to these supports.

\section{References}

[1] Futami S, Furutani A, Yoshida S. Nanometer Positioning and its Micro-dynamics, Nanotechnology, 1990;1; 31-37.

[2] Pritshow G, Philipp W, Direct Drives for High-Dynnamic Machine Tool Axes, Annual of the Cirp ;1990;39,413-16

[3] Otsuka J. Nanometer-/Subnanometer-Positioning Technology Using Feeding Mechanism, International journal of Automation Technology;2008;2;1;4-11

[4] Min-Fu Hsieha, Chin-Juei Tungb, Wu-Sung Yaoc, Meng-Chieh Wua, Yunn. Servo design of a vertical axis drive using dual linear motors for high speed electric discharge machining, International Journal of Machine Tools \& Manufacture ,2007;47;546-54

[5] Weck M, Kru"ger P, Brecher C. Limits for controller settings with electric linear direct drives ,International Journal of Machine Tools \& Manufacture ;2001;41; 65-88

[6] Moriyama S, Harada T, Takanashi A. Precision X-Y Stage with a Piezo-driven Fine-table, Bulletin of the Japan Society of Precision Engineering, 1984;50;4; 718-23 (in Japanese).

[7] Lee W C, Kim W S. An Ultraprecision Stage for Alignment of Wafers in Advanced Microlithography, Precision Engineering, 1997;21;335-45.

[8] Pahk J H, Lee S D, J. H. Park. Ultra Precision Positioning System for Servo Motor Piezo Actuator using the Dual Servo Loop and Digital Filter Implementation, International Journal of Machine Tools \& Manufacture, 2001;41,;51-63.

[9] Woronko A, Huang J. Piezoelectric Tool Actuator for Precision Machining on Conventional CNC Turning Centers, Precision Engineering, 2003;27;335-45.

[10] Elfizy T, Bone M G, Elbestwai A M. Design and Control of a Dual-stage Feed Drive, International Journal of Machine Tools \& Manufacture, 2005 ;45;153-56.

[11]Chen S J, Dwang C I .A Ballscrew Drive Mechanism with Piezo-electric Nut for Preload and Motion Control, International Journal of Machine Tools \& Manufacture;2000;40;513-26.

[12] Otsuka J, Ichikawa S, Yamaguchi Y. Development of Small Ultraprecision Displacement Small Ultraprecision Positioning Device with $1 \mathrm{~nm}$ Resolution, Journal of the Japan Society of Precision Engineering,2003; 69;10;1428-33 (in 
Japanese)..

[13] Tanaka N.Development of Nano Positioner. NSK Technical Journal, 2006;680.;29-35 (in Japanese)..

[14] Kono D, Matsubara A, Yamaji I, Fujita T. High-precision machining by measurement and compensation of motion error, International Journal of Machine Tools \& Manufacture 48;2008;1103-1110 


\section{Table}

1. Table 1 Specifications and design parameters of the dual actuated stage

\section{Figure}

1. Fig. 1. Configuration of the developed positioning system.

2. Fig. 2. Schematic view of the fine motion mechanism.

3. Fig. 3. The positioning mechanism of the fine motion: (a) Before PA expands, (b) PA expands and pushes the outer ring through the spacer, (c) The table and the ball screw move together.

4. Fig. 4. Dynamic model of the system.

5. Fig. 5. Relationship between applied voltage and PA displacement

6. Fig. 6. Experimental setup.

7. Fig. 7. Command voltage to the piezoamplifier.

8. Fig. 8. Simulated relationship between applied voltage and table displacement.

9. Fig. 9. Measured relationship between applied voltage and table displacement.

10. Fig. 10. Measured frequency response of the fine motion.

11. Fig. 11. Simulated frequency response of the coarse motion (from the motor torque to the angular velocity).

12. Fig. 12. Measured frequency response of the coarse motion (from the motor torque to the angular velocity).

13. Fig. 13. Closed loop controller for the single fine motion mechanism.

14. Fig. 14. Step response of the table: (a) step width of $1 \mu \mathrm{m}$, (b) step width of 500nm, (c) step width of $100 \mathrm{~nm}$, (d) step width of $20 \mathrm{~nm}$. 
15. Fig. 15. Step response at the tool tip point: (a) step width of $1 \mu \mathrm{m}$, (b) step width of $500 \mathrm{~nm}$, (c) step width of $100 \mathrm{~nm}$, (d) step width of 20nm.

16. Fig. 16. Block diagram of dual controller

17. Fig. 17. Results of tracking test to sinusoidal wave trajectory with amplitude of 100 $\mu \mathrm{m}$ : (a) table position and commanded trajectory by single coarse control, (b) tracking error by single coarse control, (c) table position and commanded trajectory by dual control, (d) tracking error by dual control.

18. Fig. 18. Results of tracking test to sinusoidal wave trajectory with amplitude of 500 $\mu \mathrm{m}$ : (a) table position and commanded trajectory by single coarse control, (b) tracking error by single coarse control, (c) table position and commanded trajectory by dual control, (d) tracking error by dual control. 
Table 1 Specifications and design parameters of the dual actuated stage

\begin{tabular}{c|c|c}
\hline \multicolumn{2}{c|}{ Table mass } & $5 \mathrm{~kg}$ \\
\hline \multirow{2}{*}{ Actuator } & Coarse & Ball screw (lead: $5 \mathrm{~mm}$ ) \\
\cline { 2 - 3 } & Fine & Piezoelectric actuator \\
\hline \multirow{2}{*}{ Travel } & Coarse & $200 \mathrm{~mm}$ \\
\cline { 2 - 3 } & Fine & $10 \mu \mathrm{m}$ \\
\hline \multirow{2}{*}{ Resolution } & Coarse & $65536 \mathrm{pulse} / \mathrm{rev}$ \\
\cline { 2 - 3 } & Fine & $10 \mathrm{~nm}$ \\
\hline
\end{tabular}




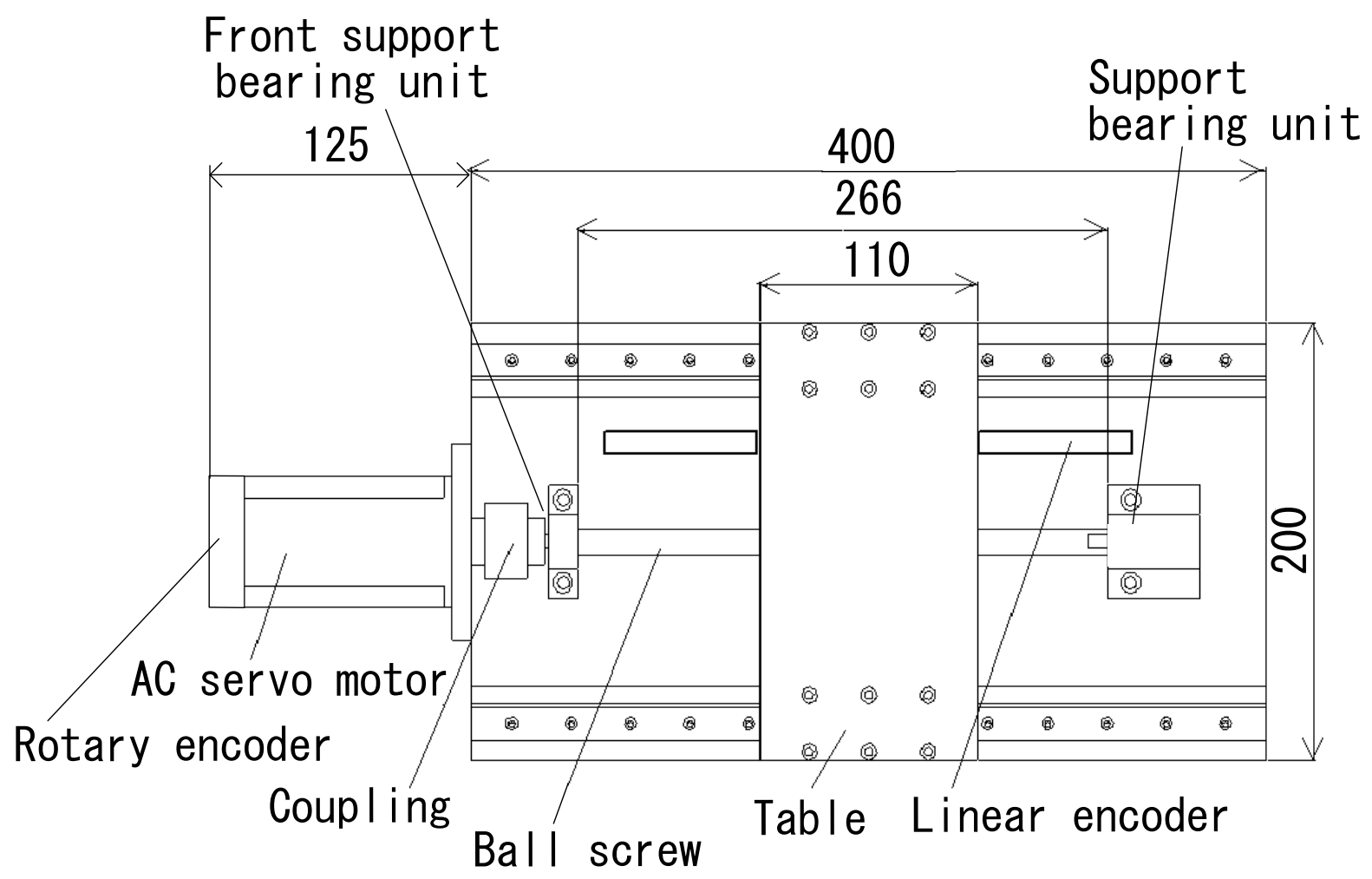

Fig. 1. Configuration of the developed positioning system. 


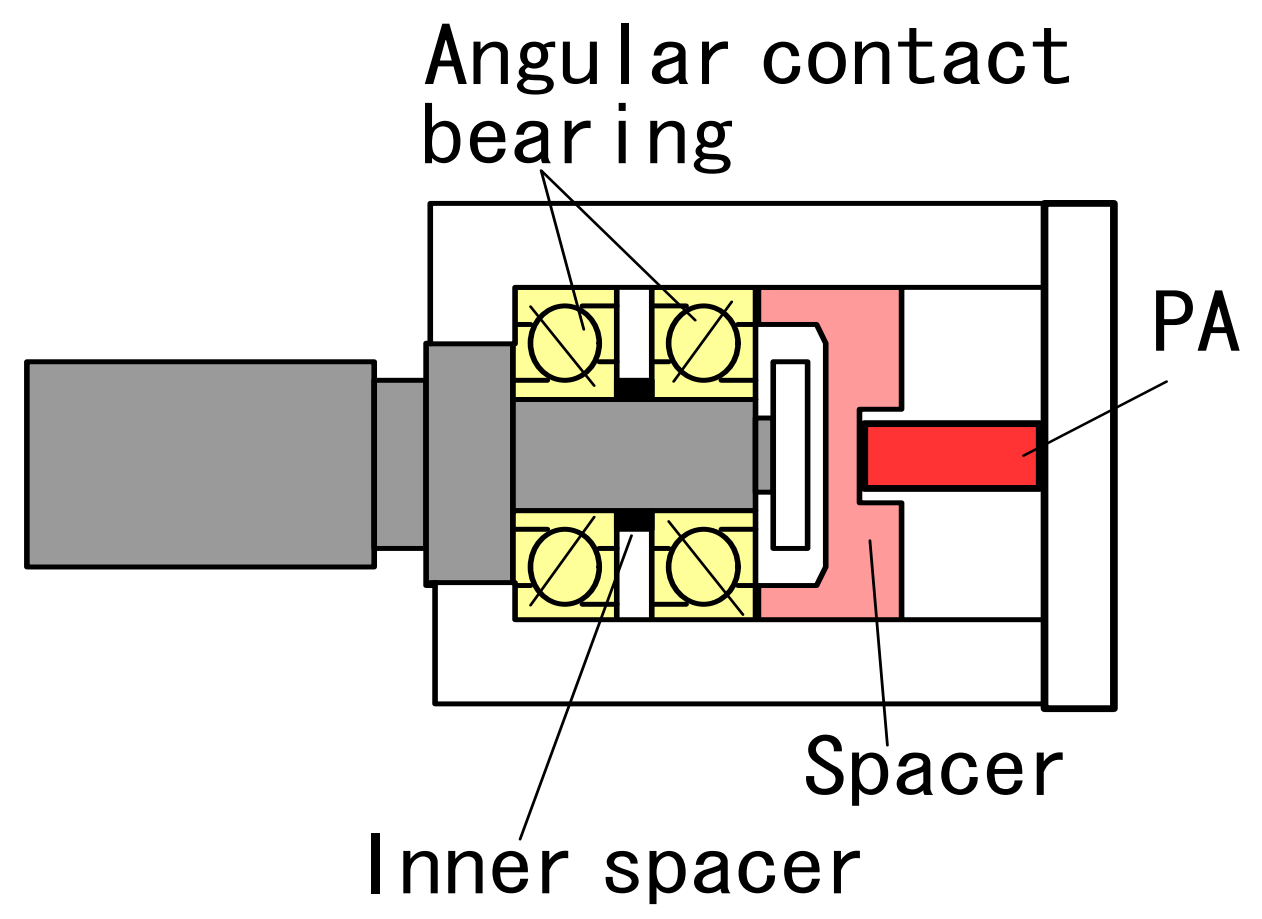

Fig. 2. Schematic view of the fine motion mechanism. 
(a)

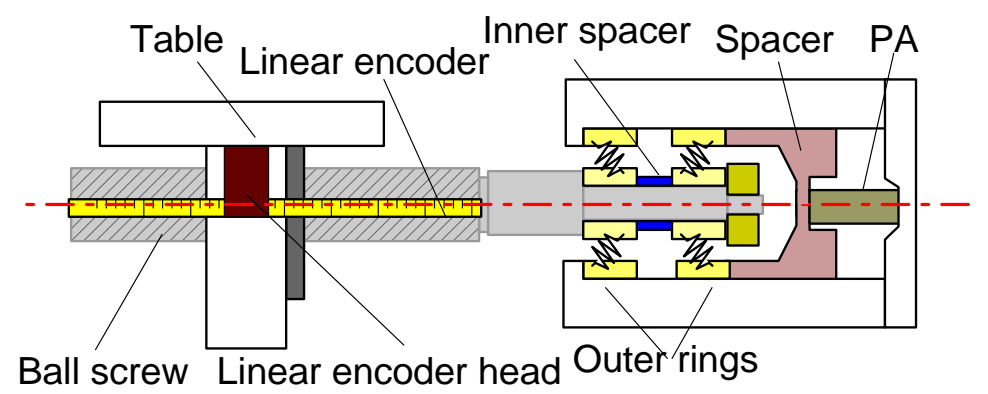

(b)

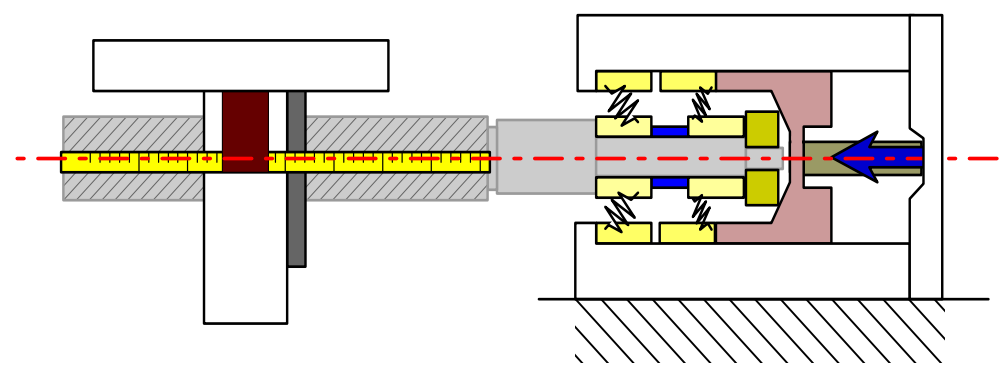

(c)

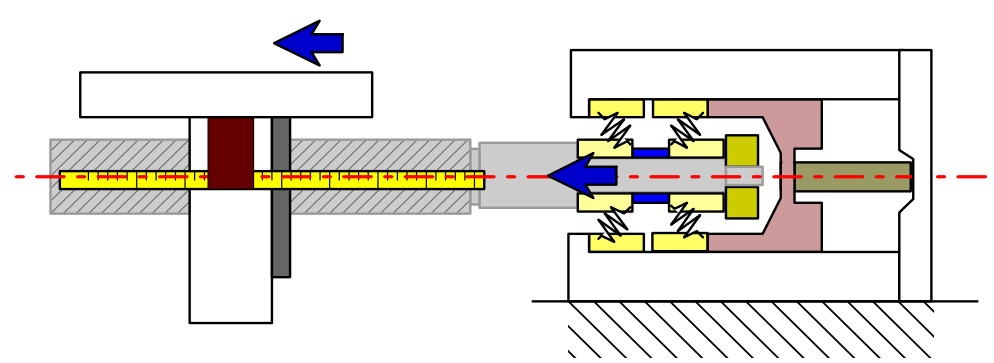

Fig. 3. The positioning mechanism of the fine motion: (a) Before PA expands, (b) PA expands and pushes the outer ring through the spacer, (c) The table and the ball screw move together. 


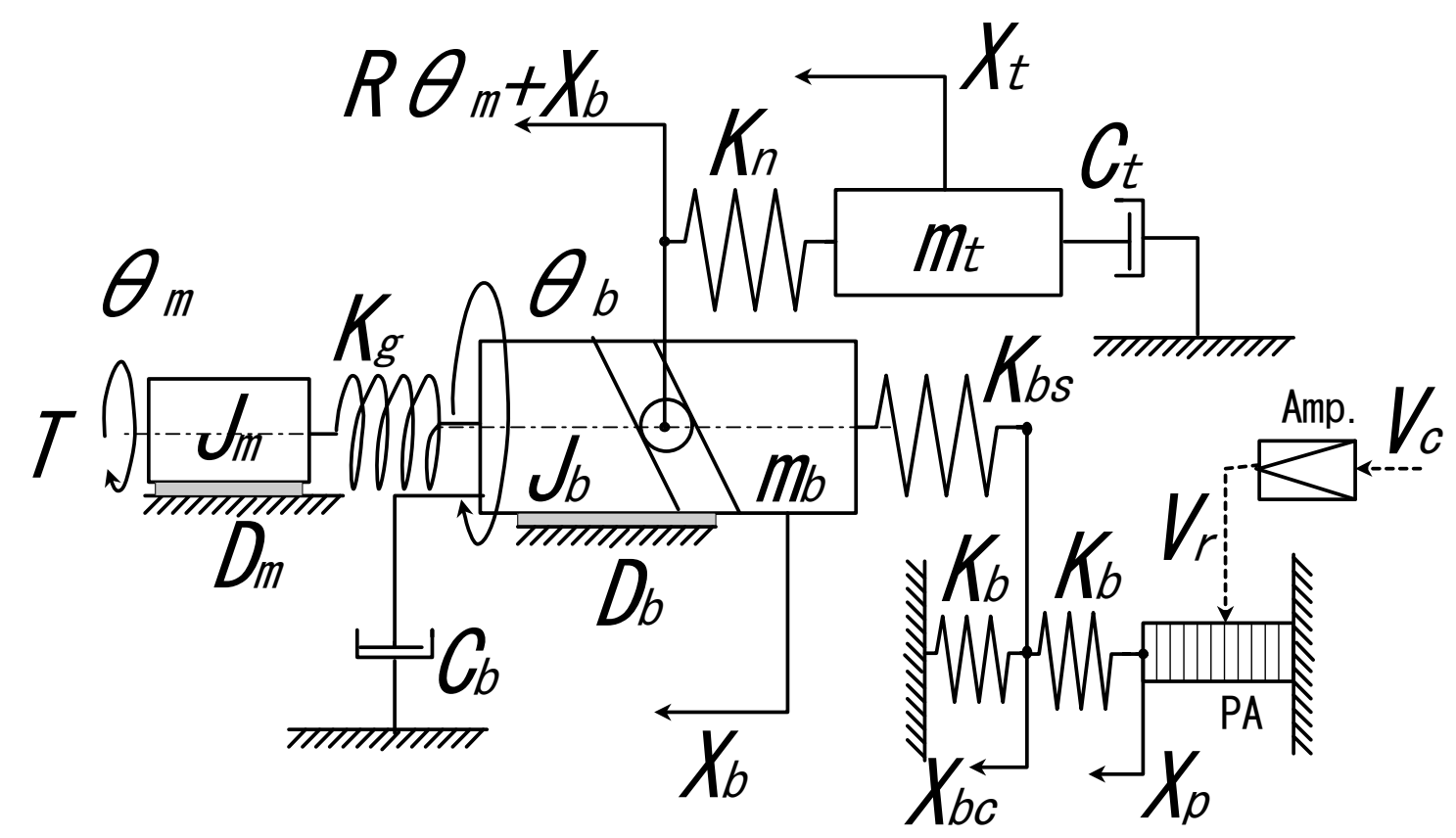

Fig. 4. Dynamic model of the system. 


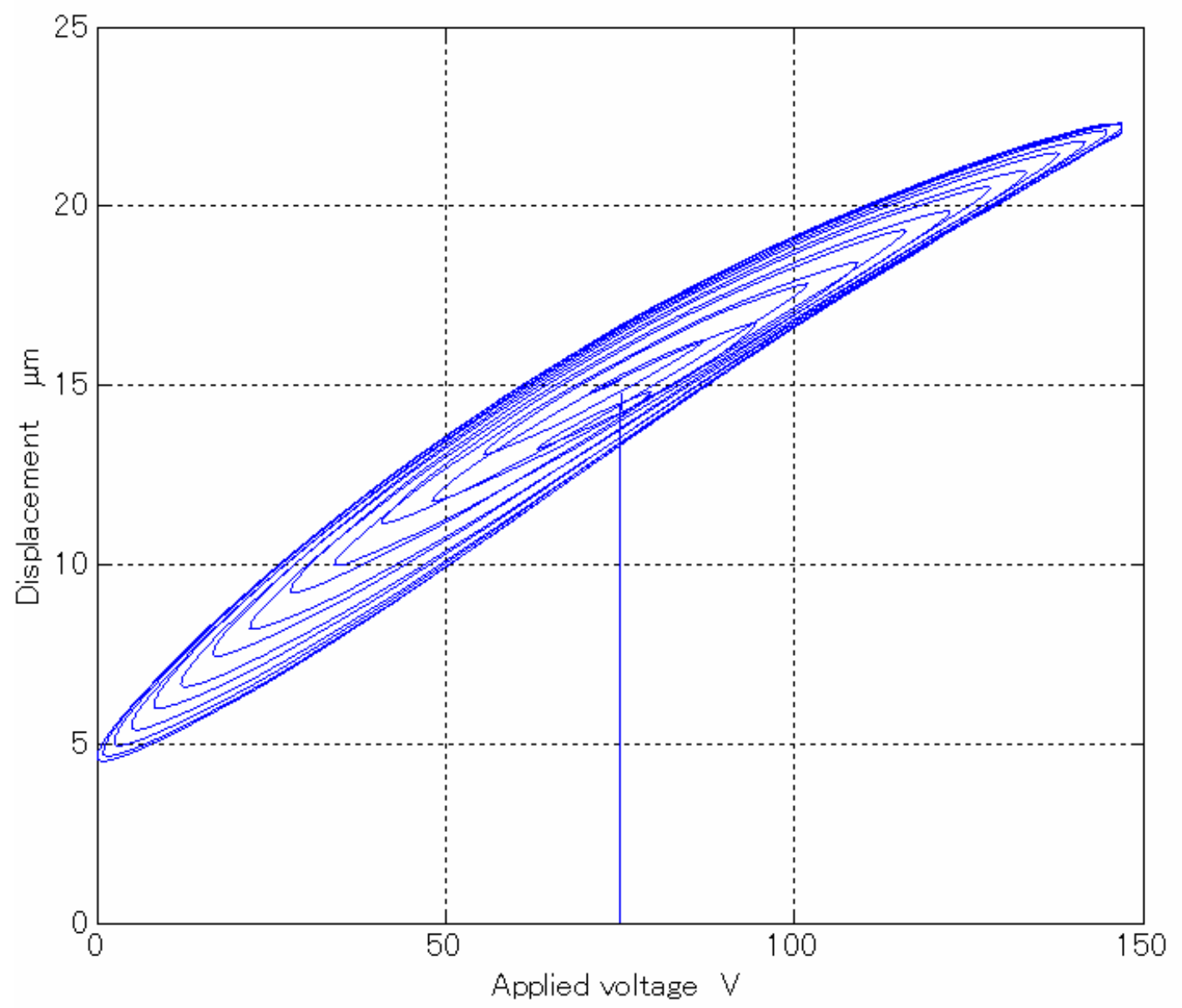

Fig. 5. Relationship between applied voltage and PA displacement 


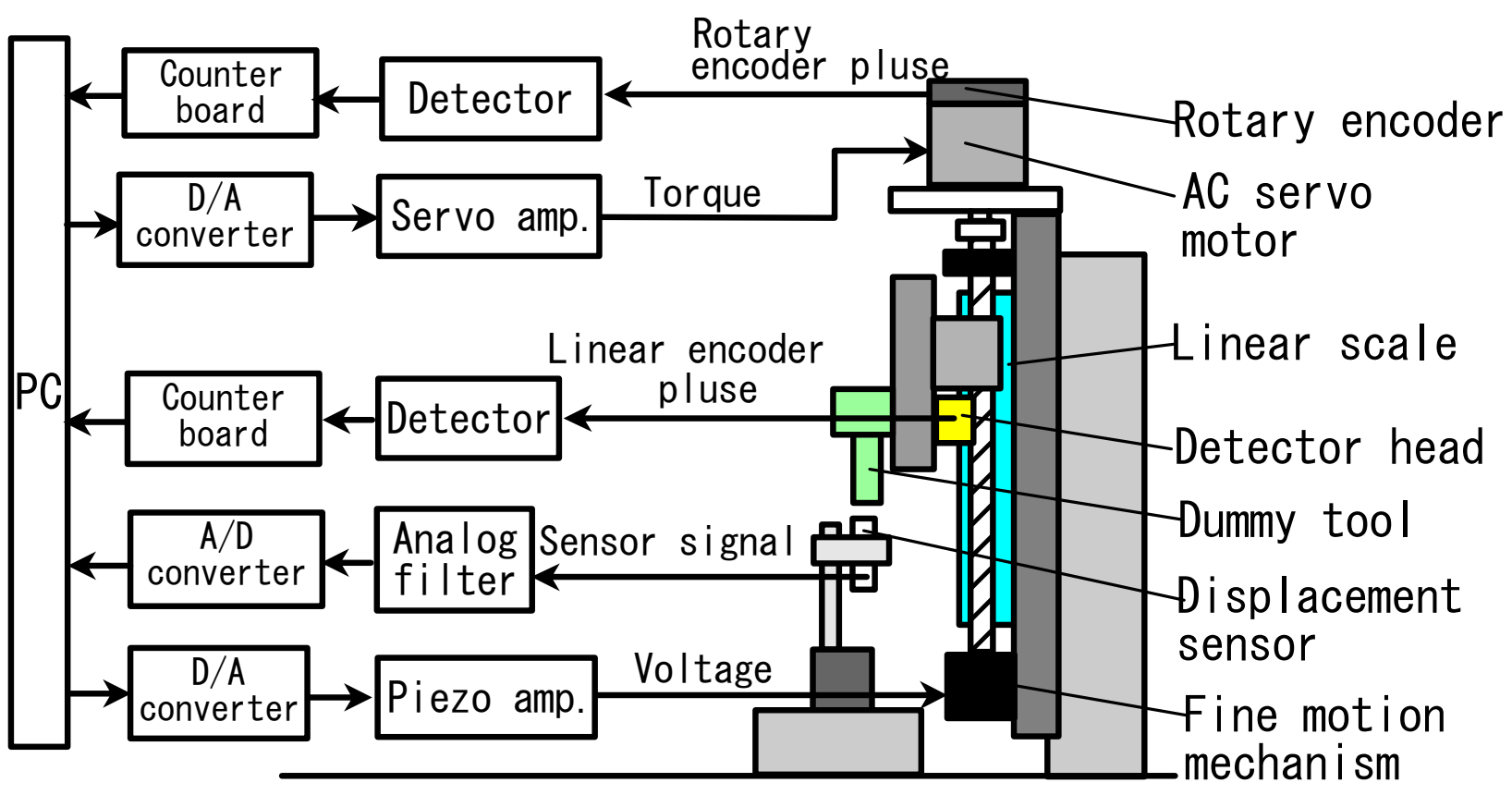

Fig. 6. Experimental setup. 


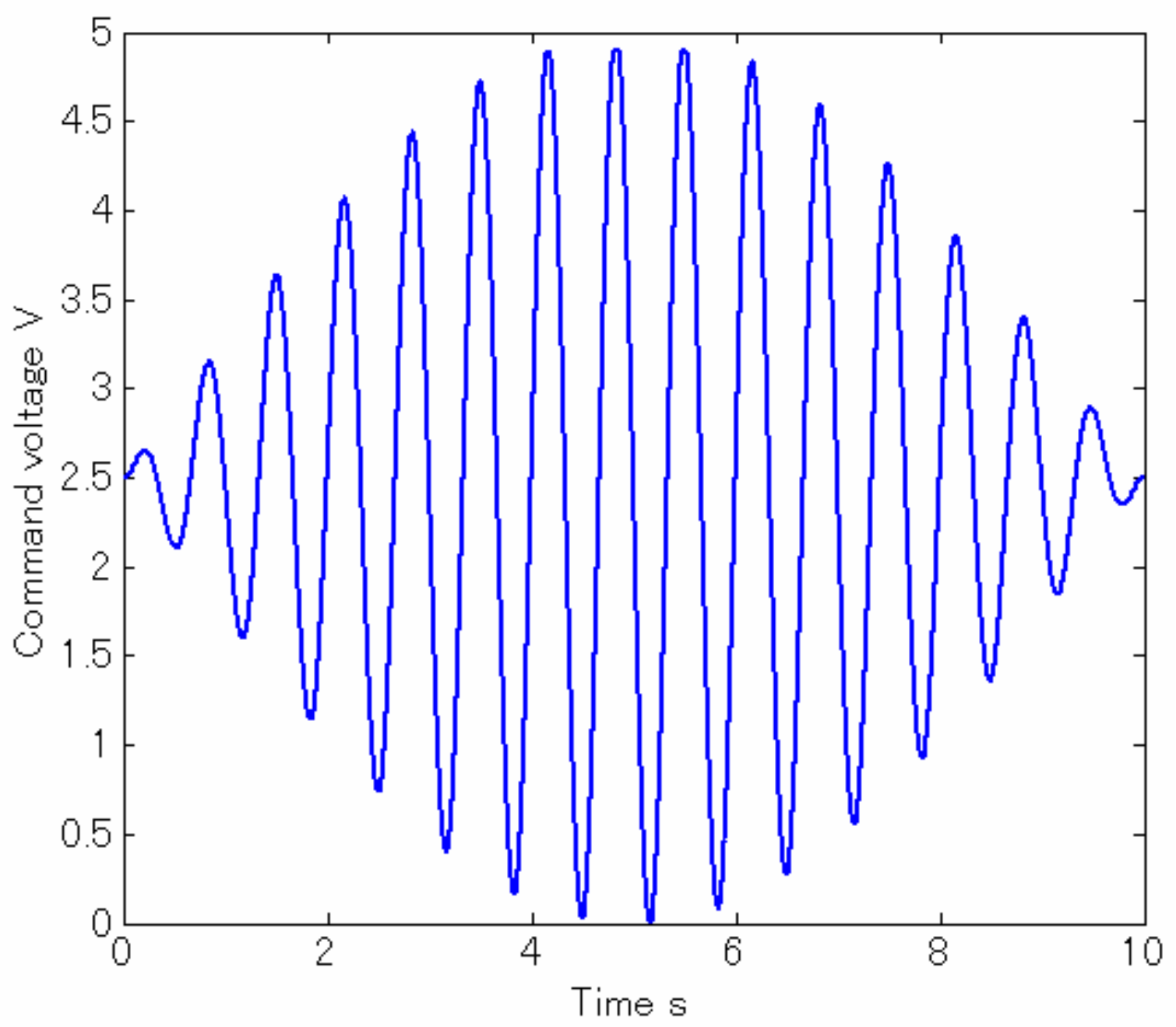

Fig. 7. Command voltage to the piezoamplifier. 


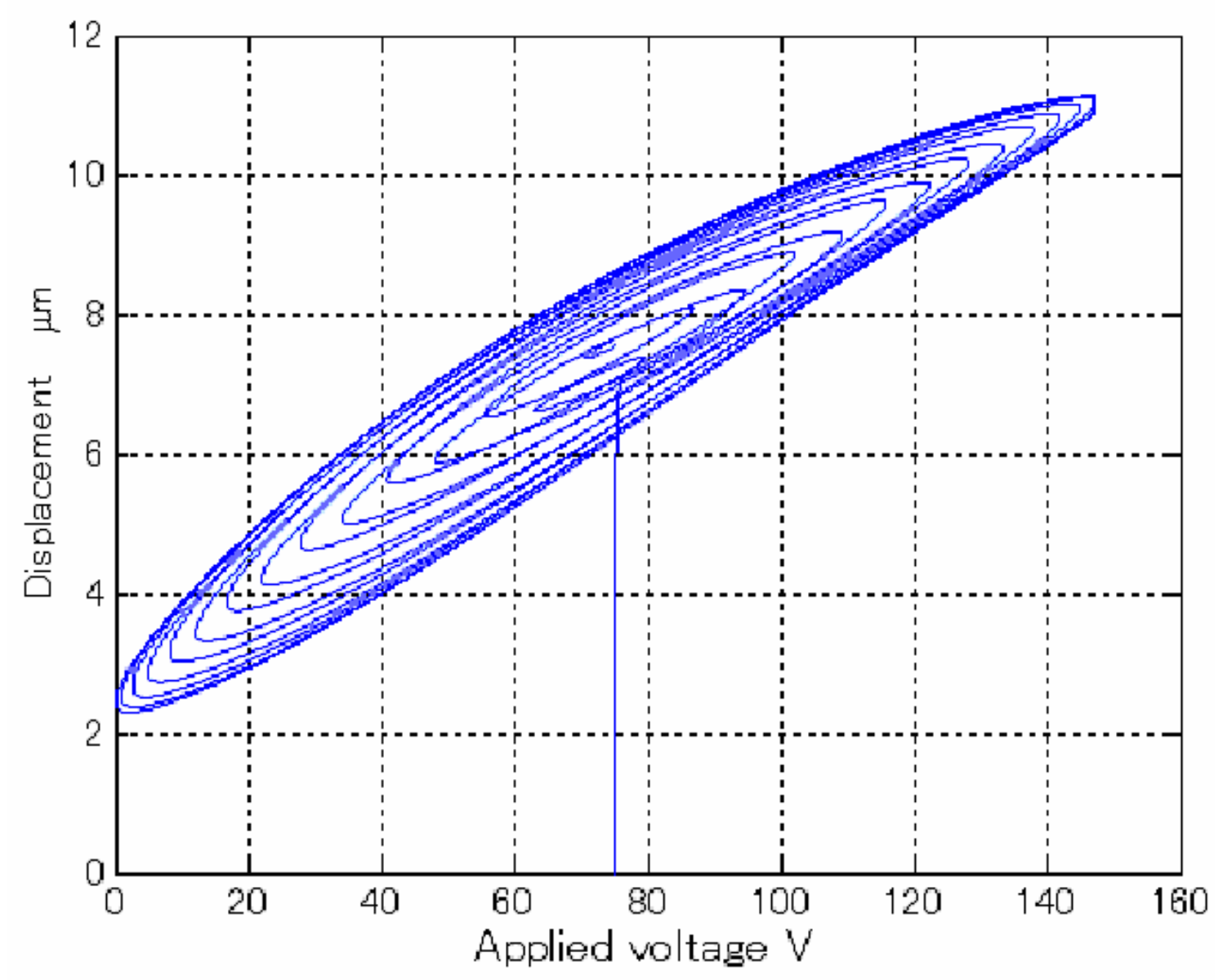

Fig. 8. Simulated relationship between applied voltage and table displacement. 


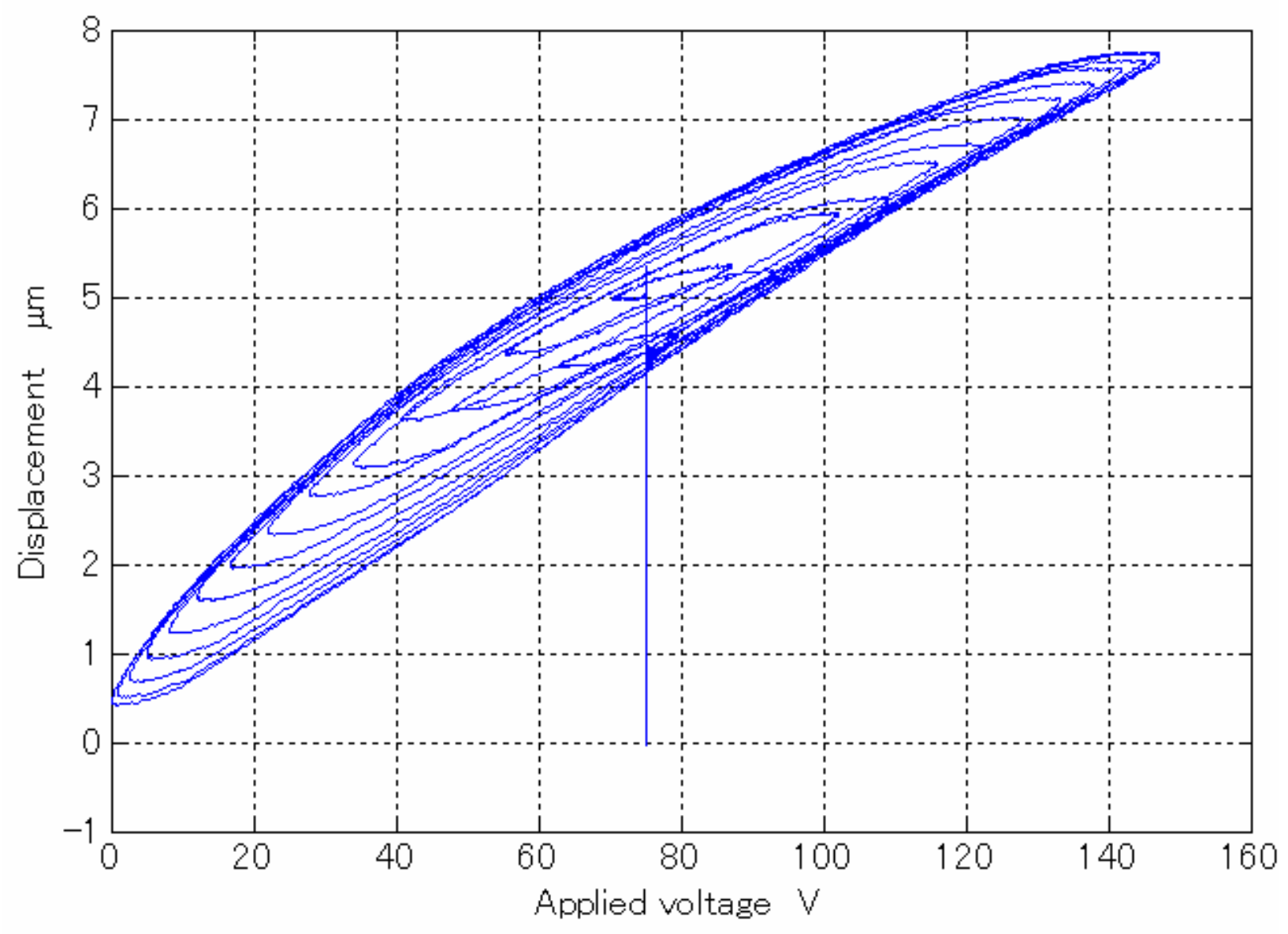

Fig. 9. Measured relationship between applied voltage and table displacement. 

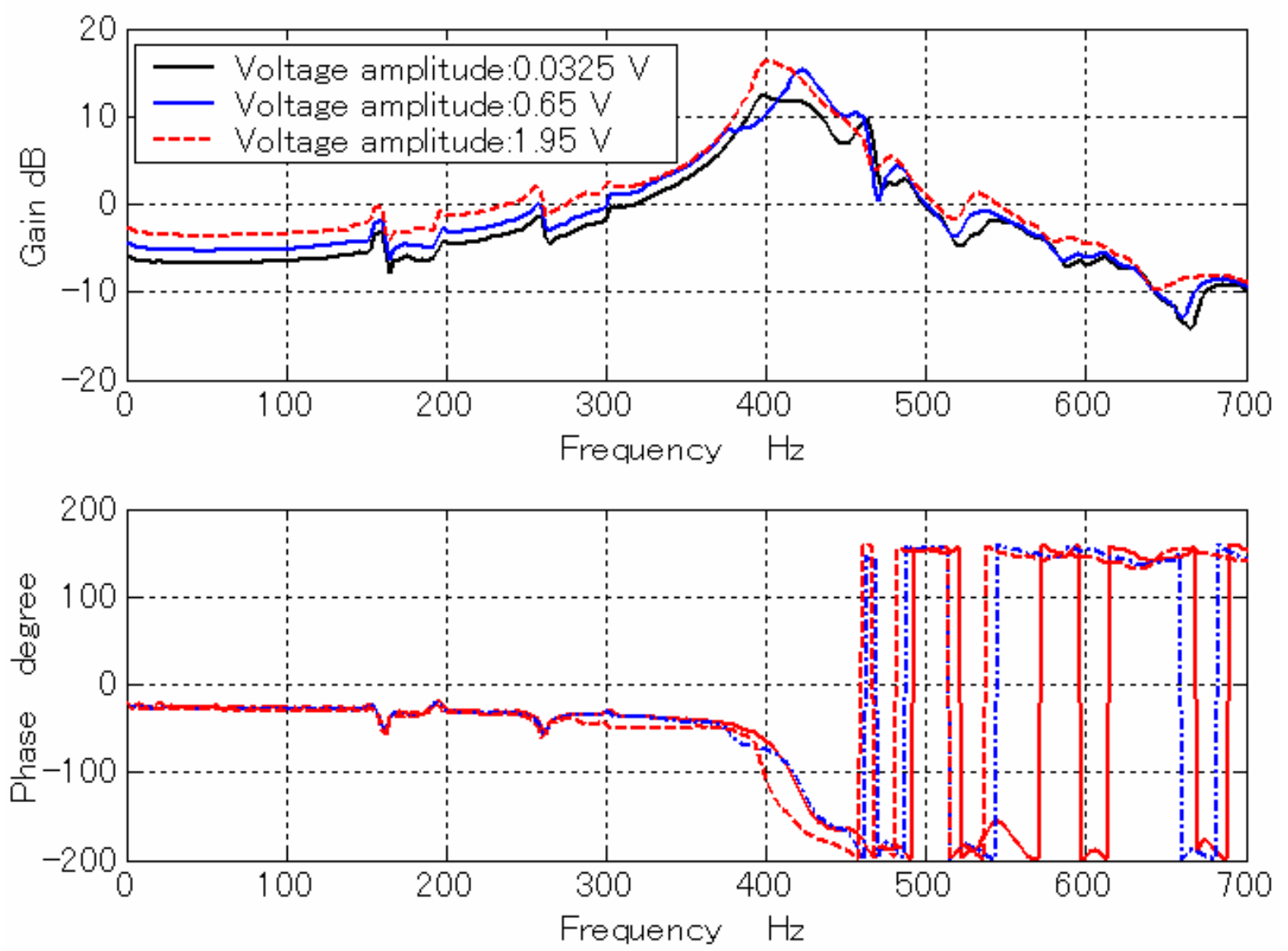

Fig. 10. Measured frequency response of the fine motion. 

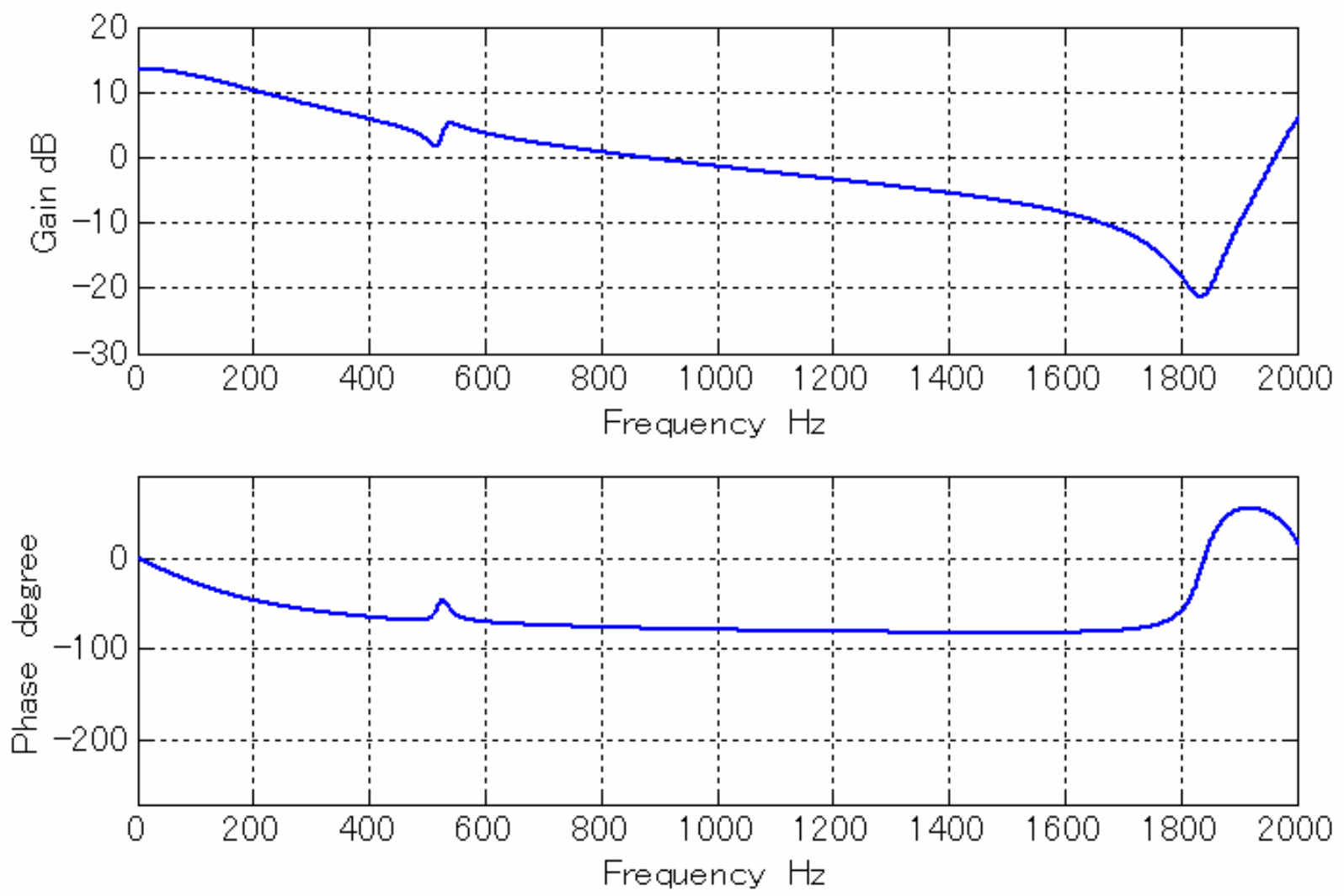

Fig. 11. Simulated frequency response of the coarse motion (from the motor torque to the angular velocity). 

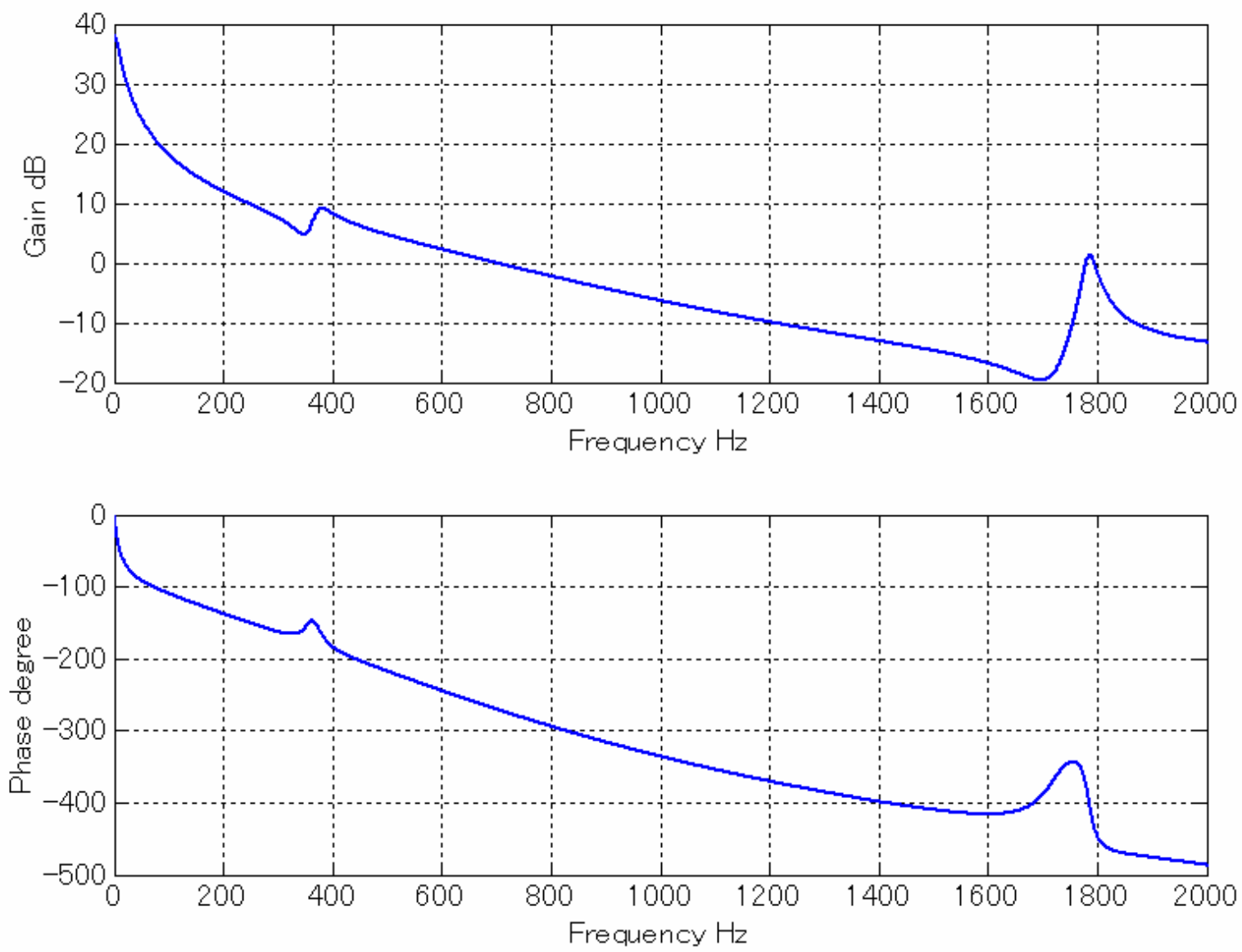

Fig. 12. Measured frequency response of the coarse motion (from the motor torque to the angular velocity). 


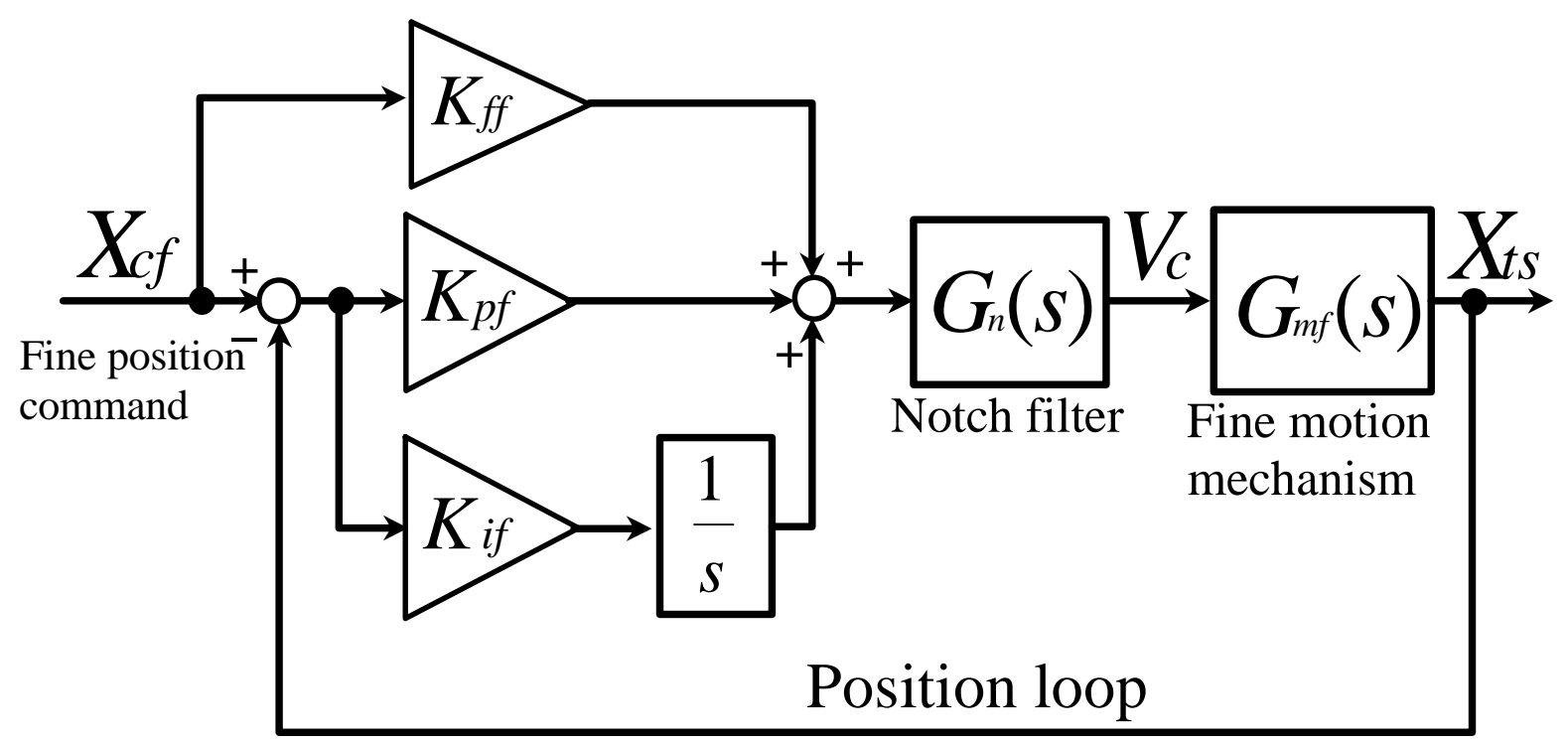

Fig. 13. Closed loop controller for the single fine motion mechanism. 

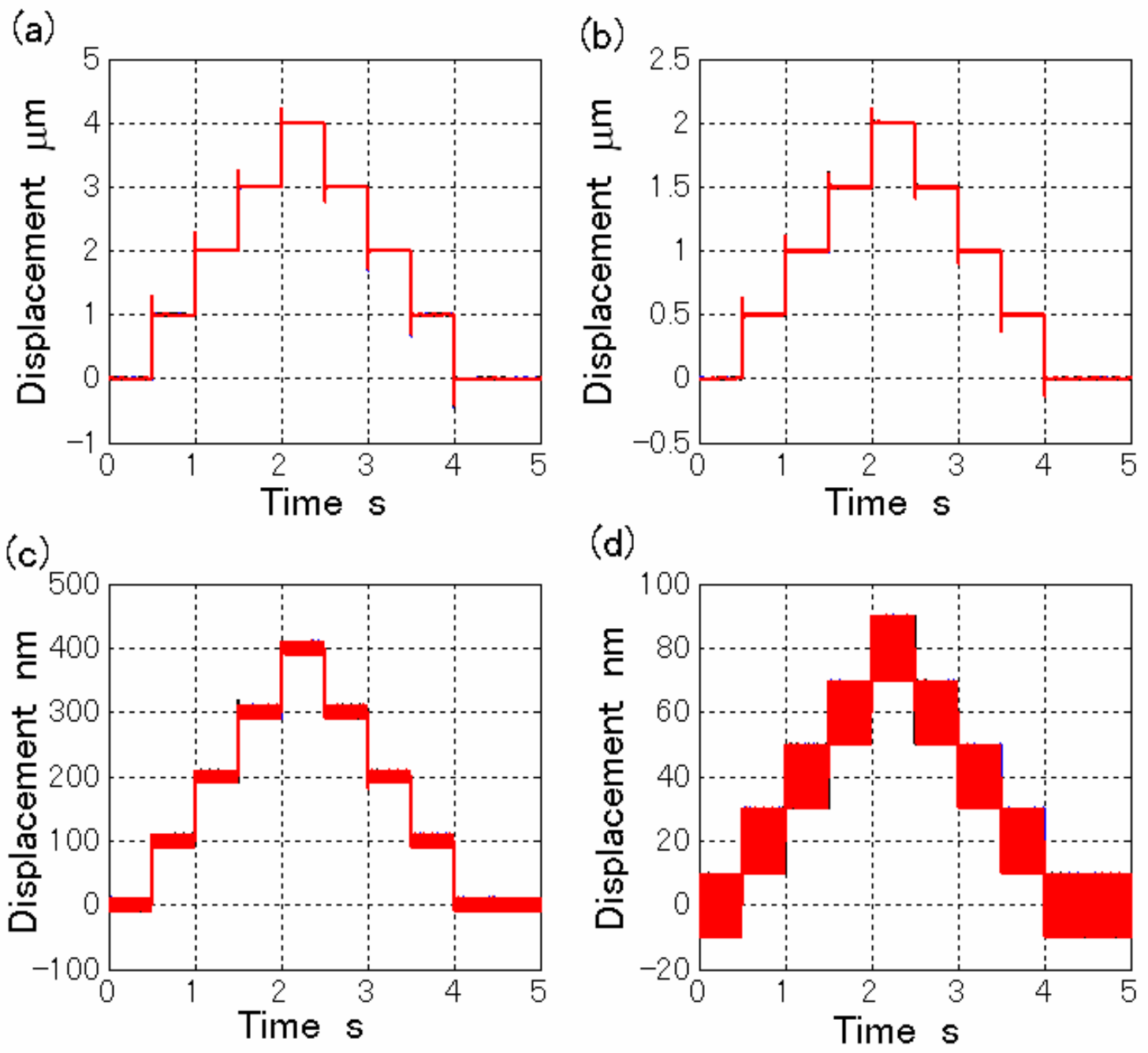

Fig. 14. Step response of the table: (a) step width of $1 \mu \mathrm{m}$, (b) step width of 500nm, (c) step width of $100 \mathrm{~nm}$, (d) step width of $20 \mathrm{~nm}$. 
(a)

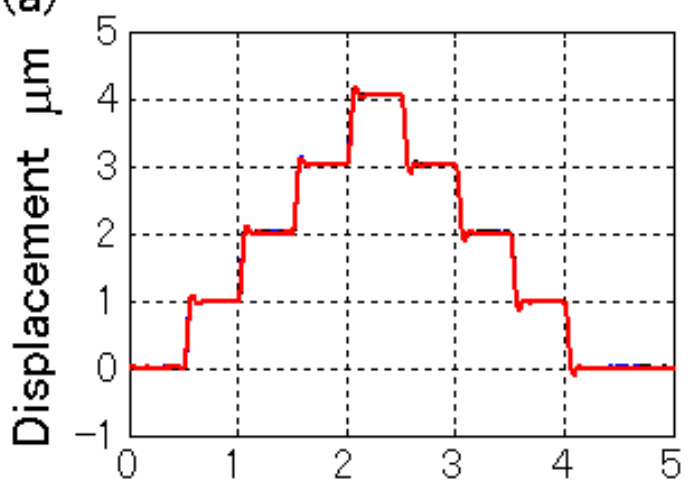

(c)

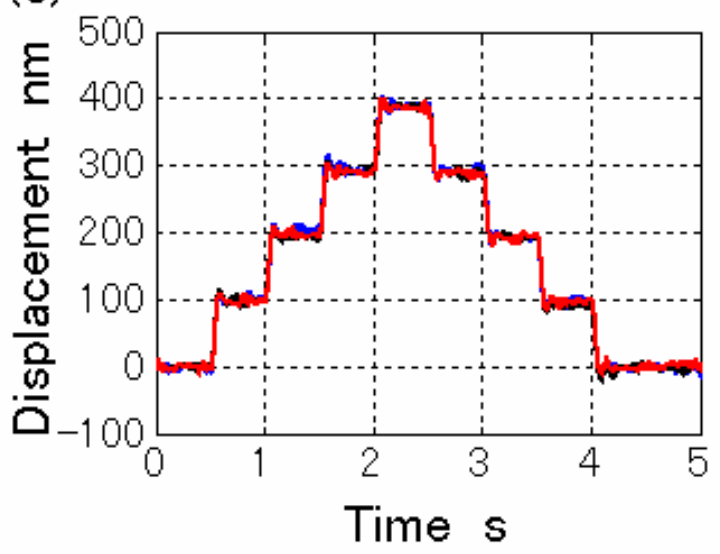

(b)

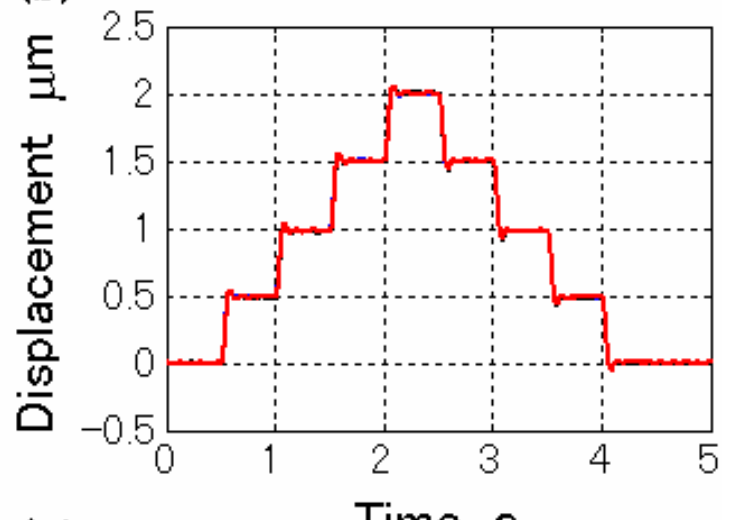

Time $s$

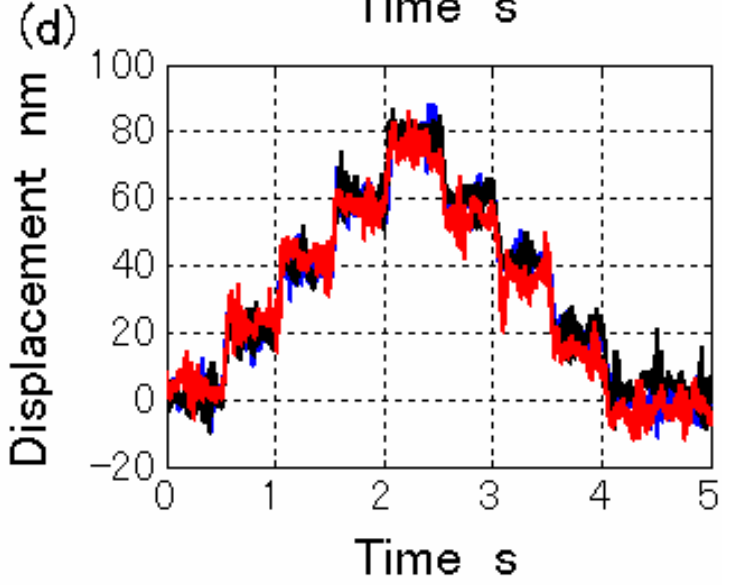

Fig. 15. Step response at the tool tip point: (a) step width of $1 \mu \mathrm{m}$, (b) step width of $500 \mathrm{~nm}$, (c) step width of $100 \mathrm{~nm}$, (d) step width of $20 \mathrm{~nm}$. 
Coarse motion control block

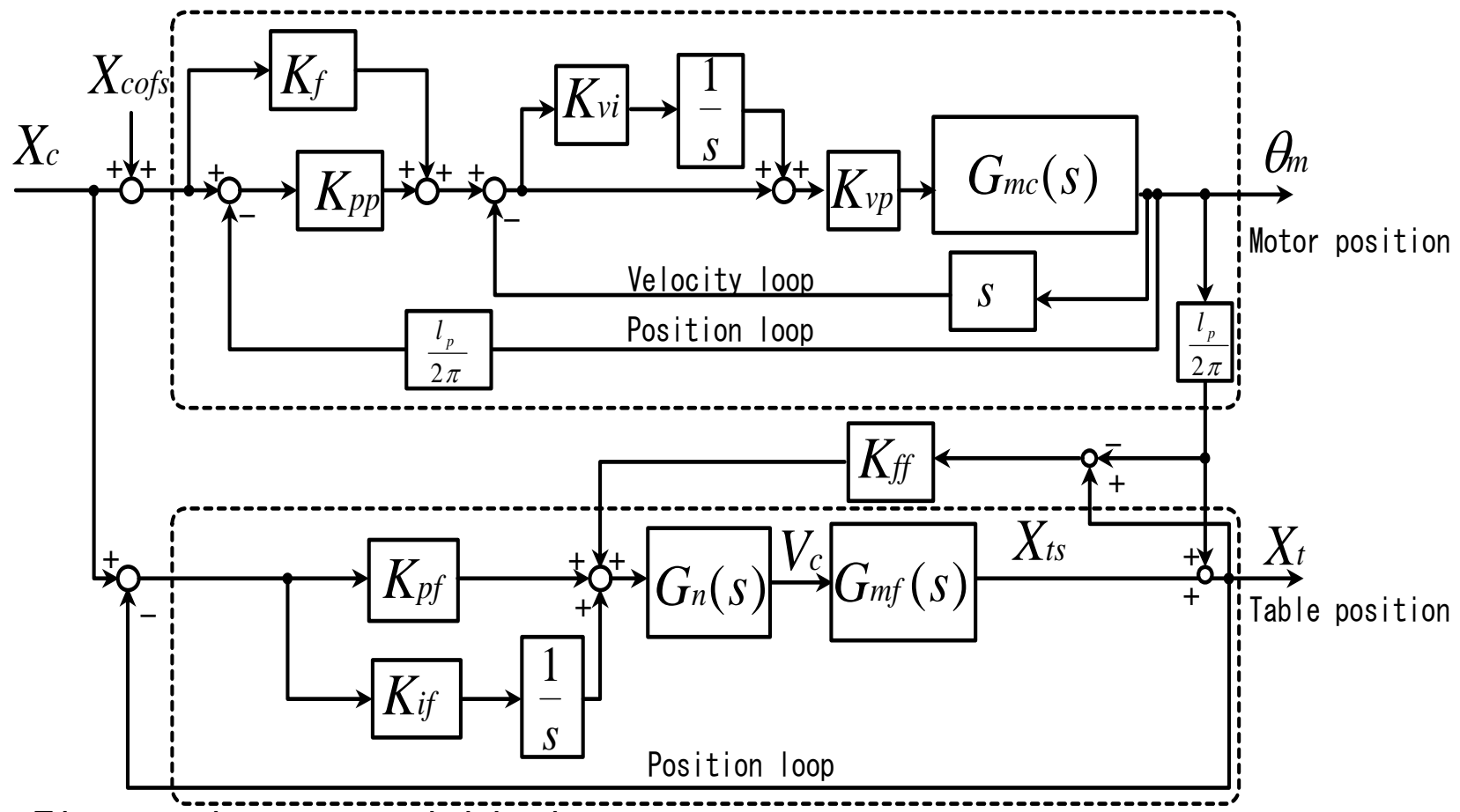

Fine motion control block

Fig. 16. Block diagram of dual controller 

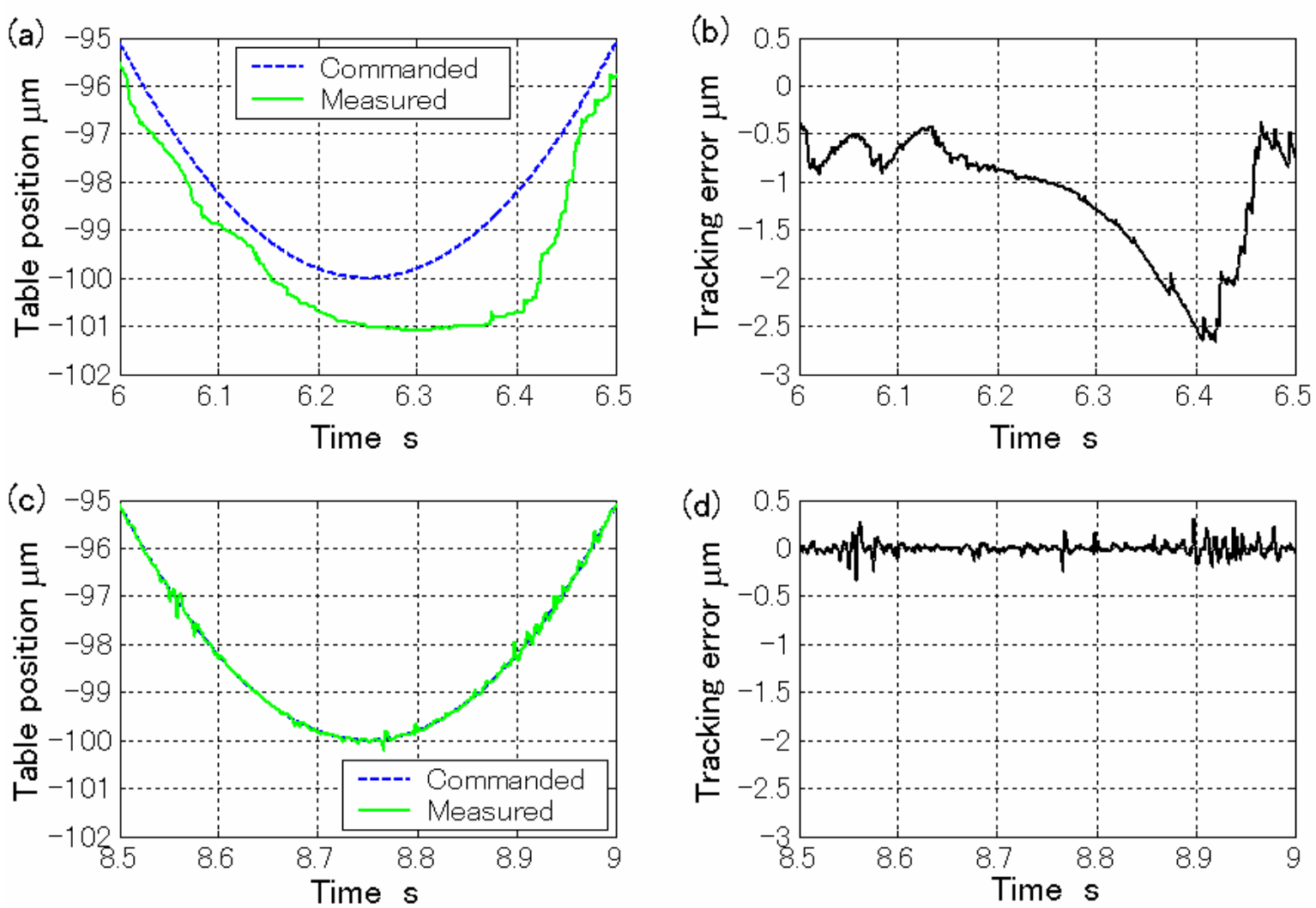

Fig. 17. Results of tracking test to sinusoidal wave trajectory with amplitude of $100 \mu \mathrm{m}$ : (a) table position and commanded trajectory by single coarse control, (b) tracking error by single coarse control, (c) table position and commanded trajectory by dual control, (d) tracking error by dual control. 
(a)

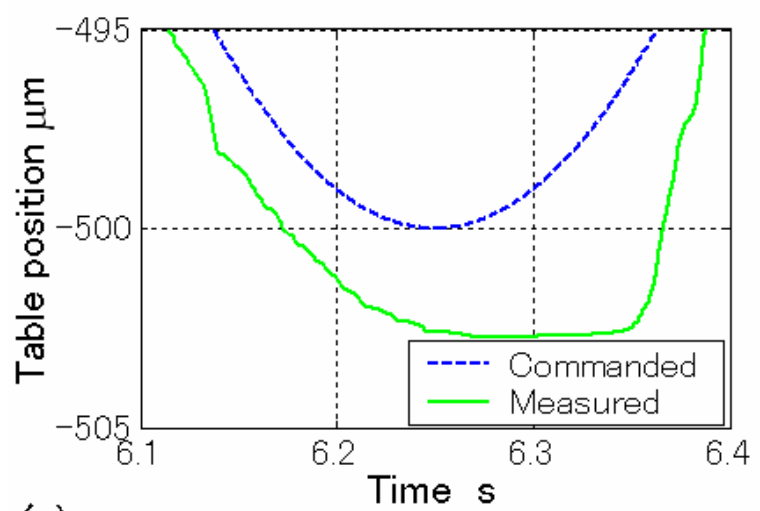

(c)

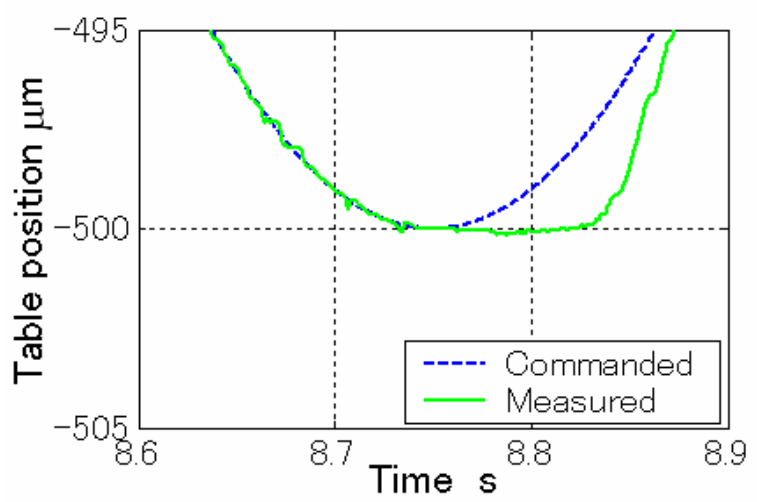

(b)

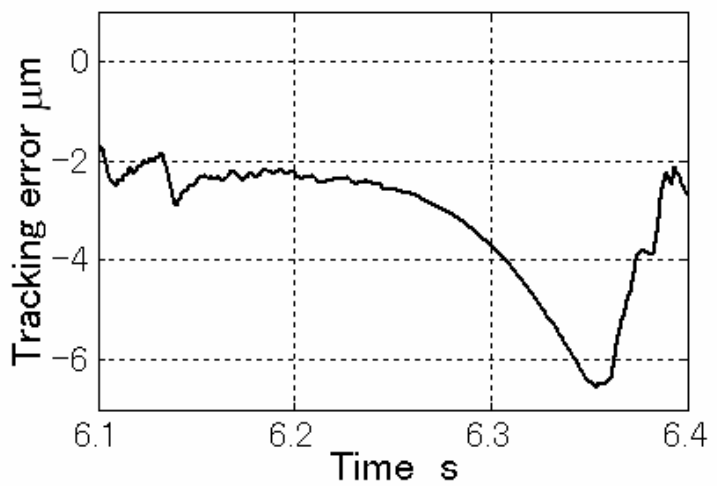

(d)

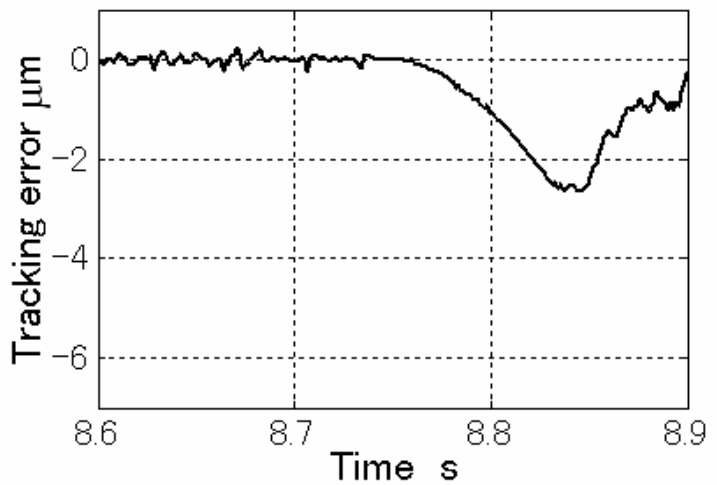

Fig. 18. Results of tracking test to sinusoidal wave trajectory with amplitude of $500 \mu \mathrm{m}$ : (a) table position and commanded trajectory by single coarse control, (b) tracking error by single coarse control, (c) table position and commanded trajectory by dual control, (d) tracking error by dual control. 